\title{
5 Índice de calidad ambiental urbana de Cuenca
}

\author{
Orellana Samaniego, María Lorena"; \\ Sellers Walden, Chester'; \\ Martínez Gavilanes, Julia ${ }^{3}$
}

Ingeniera Ambiental por la Universidad Politécnica Salesiana sede Cuenca, e investigadora en el IERSE de la Universidad del Azuay, en el área de la evaluación de la calidad ambiental en centros urbanos.

\section{María Lorena Orellana Samaniego ${ }^{1}$}

lore_orellana22@hotmail.com

Ingeniero de Sistemas por la Universidad del Azuay, Master Universitario en Gestión Sostenible de la Tierra y Territorio por la Universidad Santiago de Compostela, Magister en Geomática con mención en Ordenamiento Territorial por la Universidad del Azuay. Docente e Investigador en el IERSE de la Universidad del Azuay.

csellers@uazuay.edu.ec

Ingeniera Civil por la Universidad de Cuenca, Especialista en sistemas de información geográfica, aplicados a la gestión territorial y ambiental por la Universidad del Azuay, Master en Desarrollo Local con mención en formulación y evaluación de proyectos de desarrollo endógeno por la Universidad Politécnica Salesiana sede Cuenca. Docente e Investigadora en el IERSE de la Universidad del Azuay.

\section{Chester Sellers Walden²}

jumartinez@uazuay.edu.ec 


\section{Resumen}

Los procesos de crecimiento urbano implican, a veces, impactos negativos en el bienestar de sus habitantes. El incremento de los niveles de contaminación, la generación de residuos, el consumo excesivo de agua y energía, la disminución de áreas verdes, son algunos de los problemas que afectan a la calidad ambiental urbana. Con el objetivo de evaluar la calidad ambiental urbana de la ciudad de Cuenca se utilizó el índice de calidad ambiental-ICAU, adaptado a los valores establecidos por las normas locales. Este índice está compuesto por indicadores directos (calidad del aire, calidad del agua superficial, superficie de área verde por habitante, porcentaje de población expuesta al ruido por encima de los niveles permisibles) e indirectos (consumo residencial de agua por habitante, cantidad de residuos sólidos por habitante dispuestos en el relleno sanitario, espacio público efectivo). El estudio determinó que el puntaje de la calidad ambiental urbana para la ciudad de Cuenca es igual a 65,5 puntos, que representa una alta calidad ambiental. Sin embargo las calificaciones de los indicadores de consumo de agua por habitante y el porcentaje de la población expuesta al ruido superior a los niveles permisibles, expresan valores muy bajos. El elevado consumo de agua y el excesivo ruido generado dentro de la urbe pueden producir afecciones a la salud de sus habitantes, así como derivar en un problema a futuro por la escasez de recursos. Con este estudio se pueden desarrollar herramientas para la toma de decisiones en pro del bienestar socio-ambiental.

\section{Palabras clave:}

Indicadores ambientales, ICAU, índice ambiental.

\section{Abstract}

The processes of urban growth sometimes imply negative impacts on the weII-being of its inhabitants. Increased levels of pollution, waste generation, excessive consumption of water and energy, and the reduction of green areas are some of the problems that affect urban environmental quality. With the objective of evaluating the urban environmental quality of the city of Cuenca, the UEQI-environmental quality index was used, adapted to the values established by local regulations. This index is composed of direct indicators (air quality, surface water quality, green area per inhabitant, percentage of population exposed to noise above permissible levels) and indirect (residential consumption of water per inhabitant, amount of solid waste per inhabitant disposed in the landfill, effective public space). The study determined that the score of the urban environmental quality for the city of Cuenca is equal to 65.5 points, which represents a high environmental quality. However, the ratings of per capita water consumption indicators and the percentage of the population exposed to noise above permissible levels, express very low values. The high consumption of water and the excessive noise generated within the city can cause affections to the health of its inhabitants, as well as to derive in a problem to future by the scarcity of resources. With this study, tools can be developed for decision-making in favor of socio-environmental well-being.

\section{Keywords:}

Environmental indicators, ICAU, environmental index. 


\section{Introducción}

Con el crecimiento de las ciudades el ambiente urbano se ha transformado, en muchos casos, negativamente, debido al incremento en los niveles de contaminación, lo que afecta a la salud y el bienestar de sus habitantes (Chacón et al, 2010). Los índices tienen un papel importante en la generación de políticas públicas, lo que permite generar una percepción de los problemas con información cualitativa y cuantitativa que permita evaluar la efectividad de las decisiones públicas (Escobar, 2006).

Por la necesidad de evaluar la calidad ambiental urbana, en los últimos años se han desarrollado varios esfuerzos para obtener índices ambientales que permitan conocer la situación real de las áreas urbanas, con el fin de establecer medidas y políticas para la gestión de los territorios.

En el año 2010, con la unión de esfuerzos de actores e instituciones públicas se desarrolló el índice de calidad ambiental urbana de Cuenca, usando indicadores ambientales base que permitirían, en corto plazo, construir y evaluar las políticas ambientales, y poner en práctica acciones y estrategias que condujesen a un desarrollo sostenible a escala municipal.

Varias instituciones, a nivel nacional como regional, generan información puntual de diversas variables ambientales, estas son generalmente analizadas individualmente según las competencias de cada entidad. Este trabajo recopila información de diferentes variables ambientales con el fin de analizarlas y generar un índice de calidad ambiental urbana, que refleje la situación actual de la ciudad de Cuenca y a su vez sea utilizado como una herramienta para la toma de decisiones en pro del bienestar socio-ambiental.

\section{Métodos}

El modelo utilizó como base el índice de calidad ambiental urbana-ICAU, desarrollado por el Ministerio de Ambiente y Desarrollo Sostenible de Colombia (Díaz et al., 2013). El modelo para su aplicación fue adaptado a la ciudad de Cuenca y ha sido acoplado según la norma local.

El ICAU está compuesto por una serie de indicadores ambientales, que para su aplicación se agrupan en:

- Indicadores directos: Responsabilidad directa de la autoridad ambiental nacional

- Indicadores Indirectos: Responsabilidad de entidades territoriales, empresas de servicios públicos, entre otras.

Los indicadores son asignados dependiendo del número de población de los municipios. Cada indicador posee un valor de referencia, definidos por estándares, normas, metas y reglamentos técnicos. Su escala de calificación va de 0 a 1, en donde 0 es el peor escenario y 1 el mejor (tabla 1).

Tabla 1. Calificación de los indicadores de calidad ambiental

\begin{tabular}{|c|c|}
\hline Calificación & Categoría \\
\hline 0 & Muy bajo \\
\hline 0,3 & Bajo \\
\hline 0,5 & Medio \\
\hline 0,8 & Bueno \\
\hline 1 & Muy bueno \\
\hline
\end{tabular}

Fuente: Díaz et al., 2013 
Para el cálculo del indicador se establece que:

Indicadores directos (ID): tienen un peso mayor, $70 \%$ del total.

Indicadores indirectos (II): tienen un peso menor, $30 \%$ del total.

Cada uno de los componentes dentro de su indicador posee el mismo grado de importancia (Díaz et al., 2013), por lo que se establece que:

$$
\begin{aligned}
I D & =\sum V O I \times \frac{70}{\# \text { de indicadores del grupo reportados }} \\
I D & =\sum V O I \times \frac{70}{\# \text { de indicadores del grupo reportados }}
\end{aligned}
$$

Donde:

VOI = valor obtenido del indicador de acuerdo con la escala de calificación asignada a cada valor de referencia.

La suma de los indicadores directos e indirectos nos da como resultado el valor final del índice. Para su interpretación, el puntaje obtenido es comparado con las 5 clasificaciones de calidad ambiental urbana (tabla 2).

Tabla 2. Matriz de referencia para la asignación de la calidad ambiental urbana

\begin{tabular}{c|c|}
\hline $\begin{array}{c}\text { Calidad ambiental } \\
\text { urbana }\end{array}$ & Puntaje \\
\hline Muy baja calidad ambiental & < a 20 puntos \\
\hline Baja calidad ambiental & 20,1 a 40 puntos \\
\hline Media calidad ambiental & 40,1 a 60 puntos \\
\hline Alta calidad ambiental & 60,1 a 80 puntos \\
\hline Muy alta calidad ambiental & > a 80 puntos \\
\hline
\end{tabular}

Fuente: Díaz et al., 2013
Para la determinación del índice de calidad en la ciudad de Cuenca se utilizaron cuatro indicadores directos y tres indirectos. Estos indicadores fueron escogidos con base en los registros del año 2016.

\section{1.-Indicadores directos:}

1.1 - Superficie verde urbana por habitante: Es el número de $\mathrm{m}^{2}$ de espacios verdes urbanos por habitante. Definida por la siguiente fórmula (Díaz et al., 2013):'

$$
\mathrm{SVUH}=\frac{S V U}{\# H A B}
$$

Donde:

SVUH = Superficie verde urbana por habitante

SVU = Total de áreas verdes urbanas $\left(\mathrm{m}^{2}\right)$ \#HAB = Total de habitantes

El registro de SVU fue tomado de la matriz de clasificación de las áreas verdes del Municipio de la ciudad de Cuenca (Informe técnico, GAD municipal de Cuenca, 2014).

La estimación de \#HAB para los indicadores fue tomado de las proyecciones realizadas por el Instituto Nacional de Estadística y Censos (INEC), con base en el censo de población realizado en el año 2010. Los valores de referencias para SVUH se encuentran en la tabla 3.

1.2.- Calidad del aire: En la ciudad de Cuenca, el municipio realiza el monitoreo del aire mediante una estación de monitoreo continuo de agentes contaminantes atmosféricos, cuyo rango de cobertura es de $4 \mathrm{~km}$ de radio. Los contaminantes monitoreados son: el material particulado (PM 
Tabla 3. Matriz de referencia para la superficie verde urbana por habitante

\begin{tabular}{|c|c|}
\hline Valor de referencia & Calificación \\
\hline Menor o igual a $3 \mathrm{~m}^{2} /$ hab & 0 \\
\hline Entre $3,01 \mathrm{~m}^{2} /$ hab y $1,5 \mathrm{~m}^{2} /$ hab & 0,3 \\
\hline Entre $4,51 \mathrm{~m}^{2} / \mathrm{hab}$ y $6 \mathrm{~m}^{2} / \mathrm{hab}$ & 0,5 \\
\hline Entre $6,01 \mathrm{~m}^{2} / \mathrm{hab}$ y $7,5 \mathrm{~m}^{2} / \mathrm{hab}$ & 0,8 \\
\hline Mayor a $7,5 \mathrm{~m}^{2} / \mathrm{hab}$ & 1 \\
\hline
\end{tabular}

Fuente: Díaz et al., 2013

2,5), el monóxido de carbono (CO), el ozono (O3), el dióxido de azufre (SO2), y el dióxido de Nitrógeno (NO2). (Sellers, C., Espinoza, C. 2017)

Los datos de la red de monitoreo fueron descargados de la plataforma1. Se tomaron datos de promedios mensuales para cada uno de los contaminantes.
El índice de calidad del aire ICA toma un valor promedio generado en el periodo de tiempo consultado de cada contaminante y aplica la siguiente fórmula (Mintz, 2006):

$$
I C A=\frac{I_{H i}-I_{L o}}{B P_{H i}-B P_{L o}}\left(C_{p}-B P_{L o}\right)+I_{L o}
$$

Donde:

ICA = Índice de calidad del aire

$\mathrm{HI}=$ Valor de ICA correspondiente a BPHi

ILO = Valor del ICA correspondiente a BPLO3

$\mathrm{BPHi}=$ Punto de ruptura mayor o igual a Cp $\mathrm{BPLO}=$ Punto de ruptura menor o igual a $\mathrm{Cp}$

$\mathrm{Cp}=$ Concentración promedio observada y corregida

La figura 1 muestra los rangos de valores que son utilizados según la concentración y el tipo de contaminantes.

\begin{tabular}{|c|c|c|c|c|c|c|c|c|c|c|}
\hline & & & & $=I_{H i}-$ & $\frac{I_{L O}}{3 P_{L O}} *($ & $C_{p}-$ & $\left.B P_{L O}\right)$ & )$+I_{L}$ & Lo & \\
\hline ICA & COLOR & $\begin{array}{c}\mathrm{O} 3 \mathrm{hh} \\
\mathrm{ppb}\end{array}$ & $03 / 8 \mathrm{~h}$ & $\begin{array}{c}\text { PM2.5 } 24 \mathrm{~h} \\
u g / \mathrm{m}^{3}\end{array}$ & $\begin{array}{c}\text { PM2.5 1h } \\
u g / m 3\end{array}$ & $\left|\begin{array}{c}\text { CO } 8 \mathrm{~h} \\
\mathrm{ppm}\end{array}\right|$ & $\begin{array}{c}\mathrm{SO} 224 \mathrm{~h} \\
\mathrm{ppb}\end{array}$ & $\begin{array}{c}\mathrm{SO} 224 \mathrm{~h} \\
\mathrm{ppm}\end{array}$ & $\begin{array}{c}\mathrm{NO2} \text { 1h } \\
\mathrm{ppb}\end{array}$ & $\begin{array}{c}\mathrm{NO} 2 \mathrm{ih} \\
\mathrm{ppm}\end{array}$ \\
\hline \multirow{2}{*}{$0.5 \sigma$} & \multirow{2}{*}{ Verde } & 0,000 & 0,000 & $-0,000$ & 0,000 & 0,000 & 0,000 & 0,000 & 0,000 & 0,000 \\
\hline & & 59,000 & 0,059 & 54,000 & 15,400 & 4,400 & 34,000 & 0,034 & 0,000 & 0,000 \\
\hline \multirow{2}{*}{$51-100$} & \multirow{2}{*}{ Amarillo } & 60,000 & 0,060 & 55,000 & 15,500 & 4,500 & 35,000 & 0,035 & 0,000 & 0,000 \\
\hline & & 75,000 & 0,075 & 154,000 & 40,400 & 9,400 & 144,000 & 0,144 & 0,000 & 0,000 \\
\hline \multirow{2}{*}{$101-150$} & \multirow{2}{*}{ Naranja } & 76,000 & 0,076 & 155,000 & 40,500 & 9,500 & 145,000 & 0,145 & 0,000 & 0,000 \\
\hline & & 95,000 & 0,095 & 254,000 & 65,400 & 12,400 & 224,000 & 0,224 & 0,000 & 0,000 \\
\hline \multirow{2}{*}{$151 \cdot 200$} & \multirow{2}{*}{ Rojo } & 95,000 & 0,095 & 255,000 & 65,500 & 12,500 & 225,000 & 0,225 & 0,000 & 0,000 \\
\hline & & 115,000 & 0,115 & 354,000 & 150,400 & 15,400 & 304,000 & 0,304 & 0,000 & 0,000 \\
\hline \multirow{2}{*}{$201-300$} & \multirow{2}{*}{ Purpura } & 116,000 & 0,116 & 355,000 & 150,500 & 15,500 & \begin{tabular}{|l|}
305,000 \\
\end{tabular} & 0,305 & 650,000 & 0,650 \\
\hline & & 374,000 & 0,374 & 424,000 & 250,400 & 30,400 & 604,000 & 0,604 & 1240,000 & 1,240 \\
\hline \multirow{2}{*}{$301 \cdot 500$} & \multirow{2}{*}{ Marrón } & \multirow{2}{*}{-} & \multirow{2}{*}{-} & 425,000 & 250,500 & 30,500 & \begin{tabular}{|l|}
605,000 \\
\end{tabular} & 0,605 & 1250,000 & 1,250 \\
\hline & & & & 604,000 & 500,400 & 50,400 & 1004,000 & 1,004 & 2040,000 & 2,040 \\
\hline
\end{tabular}

Figura 1. Rangos de valores de contaminantes - Fuente: Sellers, C., Espinoza, C. 2017 
Los resultados de cada mes y de contaminante son analizados por comparación con los valores de referencia. (tabla 4)

Tabla 4. Matriz de referencia para calidad del aire

\begin{tabular}{|c|c|}
\hline Valor de referencia & Calificación \\
\hline Mayor a 300 & 0 \\
\hline Entre 201 y 300 & 0,3 \\
\hline Entre 101 y 200 & 0,5 \\
\hline Entre 51 y 100 & 0,8 \\
\hline Entre 0 y 50 & 1 \\
\hline
\end{tabular}

Fuente: Díaz et al., 2013

1.3- Porcentaje de población urbana expuesta al ruido por encima de los niveles permisibles: Es igual al porcentaje de la población expuesta a niveles de presión sonora por encima de los máximos permitidos; definida en la siguiente fórmula (Díaz et al., 2013):

$$
\text { PUAR periodo }=\frac{P U A R}{T P U} * 100
$$

Donde:

PUAR= Población expuesta al ruido por en-

cima de los límites permisibles, por periodo.

TPU=Total de la población urbana

Para determinar PUAR se parte desde el mapa de ruido de la ciudad proporcionado por el Instituto de Estudios de Régimen Seccional del Ecuador (IERSE). Se utilizaron los promedios de los horarios 07h00, 10h00, 13h00, 15h00 y $18 \mathrm{~h} 00$ para realizar el mapa de ruido diurno y el promedio horario de las 21 h00 para el mapa de ruido nocturno. Para elaborar los mapas se utilizó el programa ArcGIS 10.1, las herramientas de análisis geoestadístico IDW y base de función radial.

La información generada en los mapas, es comparada con las normas del Libro VI Anexo 5 del Texto Unificado de Legislación Secundaria del Ministerio del Ambiente (TULSMA) (TULSMA, 2015).

Se determinan las áreas que se encuentran expuestas sobre los niveles permisibles, y se establece el número de población expuesta. Los resultados obtenidos son contrastados con los valores de referencia (tabla 5).

Tabla 5. Matriz de referencia de la población expuesta al ruido

\begin{tabular}{|c|c|}
\hline Valor de referencia & Calificación \\
\hline $\begin{array}{c}\text { Mayor al } 4 \% \text { del total de } \\
\text { población urbana (cabecera) }\end{array}$ & 0 \\
\hline $\begin{array}{c}\text { Entre el 3,1 y el 4\% del total } \\
\text { de población urbana } \\
\text { (cabecera) }\end{array}$ & 0,3 \\
\hline $\begin{array}{c}\text { Entre el 2,1 y el 3\% del total } \\
\text { de población urbana } \\
\text { (cabecera) }\end{array}$ & 0,5 \\
\hline
\end{tabular}

Entre el $1,1 \%$ y el $2 \%$ del total de población urbana 0,8 (cabecera)

Menor o igual al 1\% del total de población urbana (cabecera) 1

Fuente: Díaz et al., 2013 
1.4.- Calidad del agua superficial: El índice de calidad del agua Water Quality Index (WQI) usa la suma lineal ponderada de los subíndices o la función de agregación del producto ponderado, utilizando los datos del monitoreo realizado por la Empresa Pública Municipal de Telecomunicaciones, Agua potable, Alcantarillado y Saneamiento (ETAPA) en 14 sitios, entre ríos y quebradas. Para este estudio se tomaron los 9 parámetros siguientes:

- Coliformes Fecales (en NMP/100 mL)

- $\mathrm{pH}$ (en unidades de $\mathrm{pH})$

- Demanda bioquímica de oxígeno en 5 días (DBO5 en mg/ $\mathrm{L}$ )

- $\quad$ Nitratos (NO3 en mg/L)

- $\quad$ Fosfatos (PO4 en mg/L)

- Cambio de la temperatura $\left(e{ }^{\circ} \mathrm{C}\right)$

- Turbidez (en FAU)

- $\quad$ Sólidos disueltos totales (en mg/ L)

- Oxígeno disuelto (OD en \% saturación)

El cálculo para la cualificación de la tabla 6 se realizó utilizando la calculadora virtual de la página web "Water Quality Monitoring"2. El resultado obtenido es un número que va desde 0 hasta 100, donde 0 representa una calidad muy baja y 100 excelente. Estos números son comparados con los valores de referencia para la calidad del agua superficial.
Tabla 6. Matriz de referencia de calidad del agua superficial

\begin{tabular}{|c|c|}
\hline Valor de referencia & Calificación \\
\hline Entre 0 y 25 & 0 \\
\hline Entre 25 y 50 & 0,3 \\
\hline Entre 50 y 70 & 0,5 \\
\hline Entre 70 y 90 & 0,8 \\
\hline Entre 90 y 100 & 1 \\
\hline
\end{tabular}

Fuente: Elaboración propia

2.-Indicadores indirectos:

2.1.- Consumo residencial de agua por habitante: Es el volumen de agua consumido a nivel residencial dentro de las áreas urbanas.

La calificación se realiza considerando el porcentaje de consumo con relación a la dotación neta residencial, según lo establecido en el Código Ecuatoriano de la Construcción C.E.C. Normas para estudio y diseño de sistemas de agua potable y disposición de aguas residuales para poblaciones mayores a 1000 habitantes (tabla 7). El valor obtenido es evaluado en la tabla 8.

2. www.water-research.net/index.php/water-treatment/water-monitoring/monitoring-the-quality-of-surfacewaters 
Tabla 7. Dotaciones de agua potable recomendada

\begin{tabular}{ccc}
$\begin{array}{c}\text { Población } \\
\text { (Hab) }\end{array}$ & Clima & $\begin{array}{c}\text { Dotación } \\
\text { media futura } \\
\text { (I/hab/día) }\end{array}$ \\
\hline \multirow{2}{*}{ Hasta 5000 } & Frío & $120-150$ \\
\cline { 2 - 3 } & Templado & $130-160$ \\
\cline { 2 - 3 } Cálido & $170-200$ \\
\hline 5000 a & Frío & $180-200$ \\
\cline { 2 - 3 } 50.000 & Templado & $190-220$ \\
\cline { 2 - 3 } & Cálido & $200-230$ \\
\hline \multirow{2}{*}{ Más de } & Frío & $>200$ \\
\cline { 2 - 3 } 50.000 & Templado & $>220$ \\
\cline { 2 - 3 } & Cálido & $>230$ \\
\hline
\end{tabular}

Fuente: Instituto Ecuatoriano de Normalización, 2003

Tabla 5. Matriz de referencia de la población expuesta al ruido

\begin{tabular}{|c|c|}
\hline Valor de referencia & Calificación \\
\hline $\begin{array}{c}\text { Mayor al 90\% establecido } \\
\text { para la población y clima }\end{array}$ & 0 \\
\hline
\end{tabular}

Entre el 80,1 y el 90\% del valor establecido para la pobla-

0,3 ción y clima

Entre 75 y el $80 \%$ del valor establecido para la población y clima

Entre el 70 y el $75 \%$ del valor establecido para la población y clima

Menor al 75\% valor establecido en RAS establecido para la 1 población y clima
2.2.- Cantidad de residuos sólidos por habitante dispuestos en relleno sanitario: Representa la cantidad de los residuos sólidos dispuestos en el relleno sanitario en un período de tiempo determinado. Esta información la proporcionó la Empresa Pública Municipal de Aseo de Cuenca (EMAC EP).

\section{$R S P C=\frac{R S R S}{T P U}$}

Donde:

RSPC = Cantidad de residuos sólidos por habitante dispuestos en el relleno sanitario

RSRS = Total de residuos sólidos dispuestos en el relleno sanitario (kg/año)

TPU= Total de la población urbana

El resultado es comparado con los valores de referencia (tabla 9).

Tabla 9. Valores de referencia de la cantidad de residuos sólidos

\begin{tabular}{|c|c|}
\hline Valor de referencia & Calificación \\
\hline Mayor a 1 kg/hab/día & 0 \\
\hline Entre 0,81 y $1 \mathrm{~kg} / \mathrm{hab} /$ día & 0,3 \\
\hline Entre 0,71 y 0,8 Kg/hab/día & 0,5 \\
\hline $\begin{array}{c}\text { Entre 0,61 y 0,7 Kg/hab/día } \\
\text { Menor o igual a 0,60 kg/hab/ } \\
\text { día }\end{array}$ & 0,8 \\
\hline
\end{tabular}

Fuente: Díaz et al., 2013

Fuente: Díaz et al., 2013 
2.3.- Espacio público efectivo por habitante: El indicador hace una relación entre la extensión de las zonas de espacio público efectivo y su población. Este corresponde al espacio público de carácter permanente: zonas verdes, parques, plazas y plazoletas. Los datos los proporcionó la EMAC EP.

\section{$\mathrm{EPE}=\frac{E P E U}{T P U}$}

Donde:

$E P E=$ Espacio público efectivo

EPEU = Área de espacio público efectivo en el perímetro urbano (m2)

TPU = Total de población urbana (cabecera municipal)

El resultado es comparado con los valores de referencia (tabla 10).

Tabla 10. Valores de referencia de espacio público efectivo.

\begin{tabular}{|c|c|}
\hline Valor de referencia & Calificación \\
\hline $\begin{array}{c}\text { Menor o igual a } 3,00 \mathrm{~m}^{2} / \\
\text { habitante }\end{array}$ & 0 \\
\hline $\begin{array}{c}\text { Entre } 3,01 \text { y } 4,00 \mathrm{~m}^{2} / \\
\text { habitante }\end{array}$ & 0,3 \\
\hline $\begin{array}{c}\text { Entre } 4,01 \text { y } 5,00 \mathrm{~m}^{2} / \\
\text { habitante }\end{array}$ & 0,5 \\
\hline $\begin{array}{c}\text { Entre } 5,01 \text { y } 6,00 \mathrm{~m}^{2} / \\
\text { habitante }\end{array}$ & 0,8 \\
\hline Mayor a 6,00 $\mathrm{m}^{2} /$ habitante & 1 \\
\hline
\end{tabular}

Fuente: Díaz et al., 2013

\section{Resultados}

- Áreas verdes

La superficie de área verde por habitante igual a 6,44 m²/hab, tiene una calificación de 0,8; que representa "bueno" (tabla 3).

- Calidad del aire

Los valores de PM2,5; O3, SO2, CO, $\mathrm{NO} 2$, durante todos los meses del año, indican que la calidad del aire tiene una clasificación de 1, identificada como muy buena. (tabla 4). Los resultados obtenidos se observan en la tabla 11.

- Porcentaje de población urbana expuesta al ruido por encima de los niveles permisibles

Los niveles de ruido en la ciudad, tanto diurnos como nocturnos, superan los límites permisibles, siendo los más representativos los valores registrados para el centro histórico y para la autopista de la ciudad (figura 2 y 3), lo que significa una exposición del 100\% de la población a niveles sonoros nocivos.

- Calidad del agua superficial

En los ríos se obtuvo 9 sitios con calidad buena y 3 sitios con calidad media; y en las quebradas, 1 sitio con calidad mala y 1 sitio con calidad media (figura 4). La calificación del indicador es 0,8, que representa "bueno".

habitante

- Consumo residencial de agua por

Este consumo (248 l/hab/día) es el $112,7 \%$ de lo establecido en el C.E.C. se obtiene que el indicador es mayor al 90\% establecido para la población y clima, lo que otorga una calificación de 0, que representa "muy bajo". 
Tabla 11. Índice de calidad del aire

\begin{tabular}{|c|c|c|c|c|c|}
\hline & \multicolumn{6}{c|}{ Contaminantes $\left(\mathbf{u g} / \mathbf{m}^{3}\right)$} \\
\hline Mes & PM 2,5 & $\mathbf{0 3}$ & SO2 & CO & NO2 \\
\hline Enero & 30,15 & 11,02 & 2,94 & 6,58 & -- \\
\hline Febrero & 26,46 & 9,32 & 2,94 & 8,17 & -- \\
\hline Marzo & 28,08 & 8,47 & 4,41 & 7,07 & -- \\
\hline Abril & 21,01 & 7,63 & 2,94 & 6,86 & -- \\
\hline Mayo & 32,78 & 7,63 & 4,41 & 7,83 & -- \\
\hline Junio & 30,15 & 8,47 & 4,41 & 7,86 & -- \\
\hline Julio & 35,39 & 11,86 & 4,41 & 6,90 & -- \\
\hline Agosto & 38,25 & 12,71 & 4,41 & 7,60 & -- \\
\hline Septiembre & 38,57 & 12,71 & 4,41 & 8,28 & -- \\
\hline Octubre & 31,12 & 14,41 & 2,94 & 7,51 & -- \\
\hline Noviembre & 43,63 & 15,25 & 2,94 & 6,68 & -- \\
\hline Diciembre & 32,41 & 12,71 & 2,94 & 7,08 & -- \\
\hline
\end{tabular}

- Cantidad de residuos sólidos por habitante dispuestos en relleno sanitario

Los habitantes, en promedio, producen $0,542 \mathrm{~kg}$ de residuos sólidos diarios. Este indicador obtuvo una calificación de 1, que representa "muy bueno".
- Espacio público efectivo por habitante

Cuenca posee un espacio público efectivo por habitante de 9,7 m2/hab, lo cual comparando con los valores de referencia del índice del indicador, posee una calificación de 1, que representa "muy bueno".

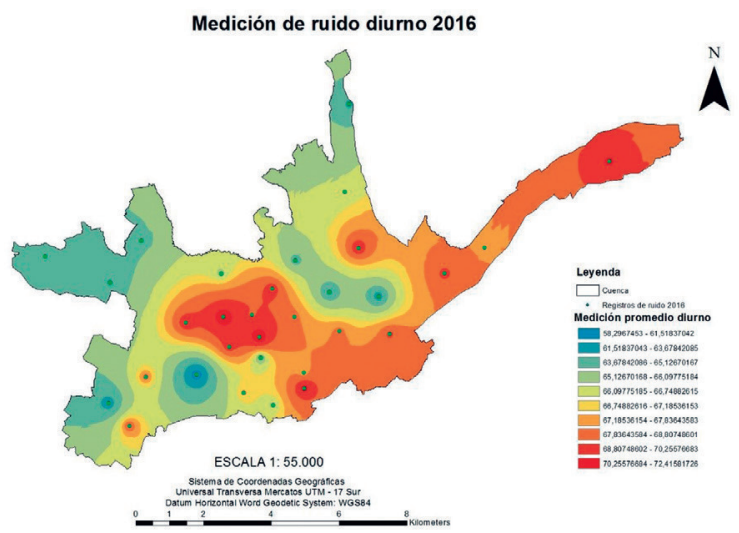

Figura 2. Mapa de ruido diurno de la ciudad de Cuenca, año 2016 


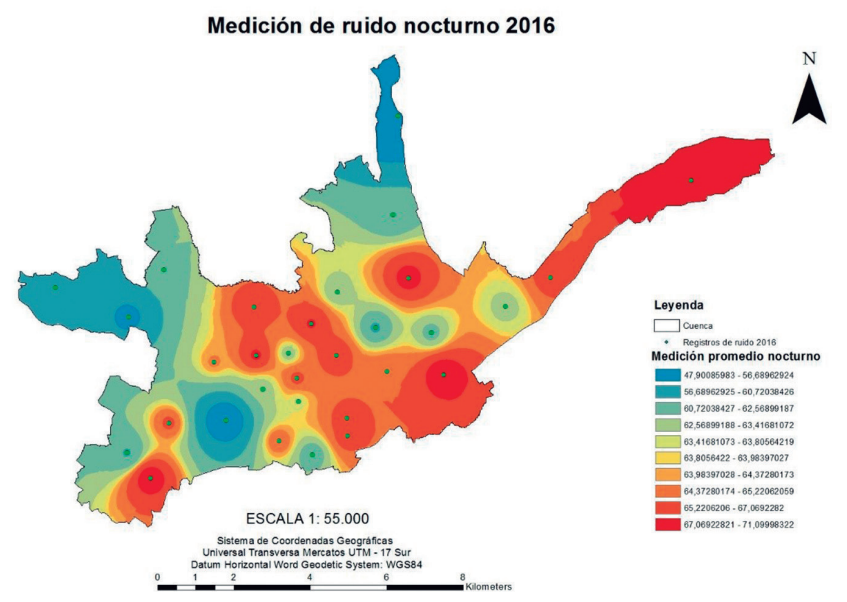

Figura 3. Mapa de ruido nocturno de la ciudad de Cuenca, año 2016

- Índice de calidad ambiental

Como resultado de la suma de los indicadores directos e indirectos, se obtiene un valor de 65,5 puntos, que comparando con los valores de referencia para la asignación de la calidad ambiental urbana, se considera que la ciudad de Cuenca presenta una alta calidad ambiental (tabla 12).

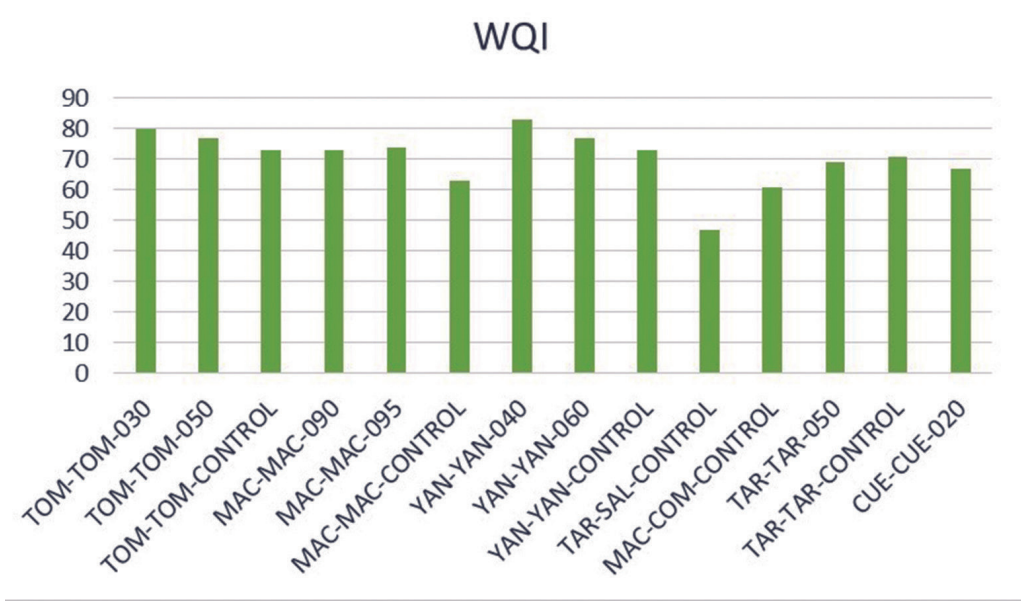

Figura 4. Calidad del agua en puntos de muestreo de la ciudad de Cuenca, año 2016 


\section{Conclusiones}

Cinco de los indicadores utilizados para evaluar la calidad ambiental en la ciudad de Cuenca presentan valores permisibles (clasificados como bueno y muy bueno). Por otro lado dos indicadores expresan una baja calidad ambiental (muy bajo). En términos generales el área urbana de Cuenca presenta una buena calidad ambiental.

El indicador del porcentaje de la población expuesta al ruido superior a los límites permisibles, es el más preocupante porque significa que un $100 \%$ de la población está expuesta a valores por sobre lo permitido en la legislación ambiental. Este tipo de exposiciones generan afecciones de diversa índole en la población, destacándo- se efectos sobre la audición, trastorno del sueño y reposo, efectos psicofisiológicos, sobre la salud mental y el rendimiento, y efectos en el comportamiento. (Berglund, el at., 1999)

Otro indicador particular es el abuso en el consumo de agua por los habitantes, siendo del $112.7 \%$ de lo establecido en el C.E.C. Entre las actividades principales asociadas al exagerado consumo de este recurso destacan el lavado de vehículos, riego de jardines, fugas de agua que no son controladas, llaves abiertas en los hogares e instituciones educativas, hidrantes en uso, baños prolongados en los hogares. En 2012 existe entre un $40 \%$ y $60 \%$ de desperdicio de agua potable. (El Telégrafo, 2012)

Tabla 12. Matriz de resultados del indicador ambiental urbano para Cuenca

\begin{tabular}{|c|c|c|c|}
\hline & Indicador & Resultado & Calificación \\
\hline & $\begin{array}{l}\text { Superficie de área verde por } \\
\text { habitante }\end{array}$ & 6,4 m3/hab & 0,8 \\
\hline & Calidad del aire & Entre 0 y 50 & 1 \\
\hline d & Calidad de agua superficial & Entre 70 y 90 & 0,8 \\
\hline & $\begin{array}{l}\text { Porcentaje de población urbana expuesta al } \\
\text { ruido por encima de los niveles permisibles }\end{array}$ & $\begin{array}{l}\text { > al 4\% de la } \\
\text { población urbana }\end{array}$ & 0 \\
\hline & Valor total del indicador directo & 45,5 & \\
\hline & Consumo residencial de agua por habitante & 248 I/hab/día & 0 \\
\hline 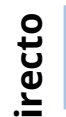 & $\begin{array}{c}\text { Cantidad de residuos sólidos por habitante } \\
\text { dispuestos en el relleno sanitario }\end{array}$ & 0,54 & 1 \\
\hline 흥 & $\begin{array}{c}\text { Espacio público efectivo por } \\
\text { habitante }\end{array}$ & 9,7 & 1 \\
\hline & Valor total del indicador indirecto & 20 & \\
\hline & ndicador de calidad ambiental Cuenca & 65,5 & \\
\hline
\end{tabular}


Es indispensable desarrollar un diseño de muestreo y monitoreo en la ciudad, haciendo énfasis en los indicadores que en este estudio no han sido utilizados. Monitoreo en donde las instituciones encargadas puedan reportar periódicamente, para establecer una metodología que pueda ser utilizada en los próximos años. Por falta de información para este estudio se utilizaron únicamente siete de los 14 indicadores considerados para municipios con población urbana entre los 100.000 y 499.999 habitantes.

\section{Referencias}

Berglund, B., Lindvall, T., Schwela, D. H., \& others. (1999). Guías para el ruido urbano. Centro Panamericano de Ingeniería Sanitaria Y Ciencias Del Ambiente, OPS/CEPIS. Recuperado de https://editorial.cda. ulpgc.es/ftp/Instalaciones2/ACUSTICA/ ANEXO/1Comoidad/OMSalud-Guias\%20 para\%20el\%20Ruido\%20Urbano.pdf

Chacón, R. M., \& Ornés, S. (2010). La calidad ambiental urbana: construcción de un sueño ciudadano. In Anales de la Universidad Metropolitana (Vol. 10, pp. 229-246). Universidad Metropolitana. Recuperado de https://dialnet.unirioja.es/ servlet/articulo?codigo=3625183

Díaz, A., Granados, S., \& Valdés, D. (2013). ÍNDICE DE CALIDAD AMBIENTAL URBANAICAU (p. 112). Colombia: Ministerio del Ambiente y Desarrollo Sostenible.

El Telégrafo. (2012, de agosto). El desperdicio de agua en Cuenca llega al 60\%. Recuperado de http://www.eltelegrafo.com.ec/ noticias/regional-sur/1/el-desperdiciode-agua-en-cuenca-llega-al-60
Escobar, L. (2006). Indicadores sintéticos de calidad ambiental: un modelo general para grandes zonas urbanas. Eure (Santiago), 32(96), 73-98.

Instituto Ecuatoriano de Normalización. (2003). NORMAS PARA ESTUDIO Y DISEÑO DE SISTEMAS DE AGUA POTABLE $Y$ DISPOSICIÓN DE AGUAS RESIDUALES PARA POBLACIONES MAYORES A 1000 HABITANTES.

Ministerio del Ambiente (2003). Libro VI Anexo 5 De la Calidad Ambiental. En Texto Unificado de Legislación Ambiental (pág. 13). Quito.

Mintz, D. (2006). Guideline for Reporting of Daily Air- Air Quality Index (AQI). Carolina del Norte : Enviromental Protection Agency.

Sellers, C., Espinoza, C. (2017). Publicación de los contaminates atmosféricos de la estación de monitoreo en tiempo real de la ciudad de Cuenca, utilizando servicios estándares OGC. ACl Avances en Ciencias e Ingenerias, 9(15), 